\title{
Darwin's Theorem Revised: Survival of The Careerist
}

\author{
Robert Skopec* \\ Researcher analyst, Slovakia
}

*Corresponding author: Robert Skopec, Researcher analyst, Dubnik, Slovakia, Europe.

Received Date: February 26, 2019

Published Date: March 25, 2019

\begin{abstract}
Psychopathy of the careerist is a developmental disorder marked by emotional deficits and an increased risk for antisocial behavior. It is not equivalent to the diagnosis Antisocial Personality Disorder, which concentrates only on the increased risk for antisocial behavior and not a specific cause-i.e., the reduced empathy and guilt that constitutes the emotional deficit. Our review considers data regarding the neurobiology of this disorder. Dysfunction within the amygdala's role in reinforcement learning and the role of ventromedial frontal cortex in the representation of reinforcement value is stressed. Data is also presented indicating potential difficulties within parts of temporal and posterior cingulate cortex. Suggestions are made with respect to why these deficits lead to the development of the disorder. Knowledge of recent neurobiology is proving our thesis that Darwin was wrong when formulated his theorem „Survival of the fittest “. Reality in 21 centuries is showing that "Survival of the careerist "based on the Quantum Entanglement Entropy (QEE) is more valid principle of Social Dynamics in our days. Careeristic Competition is the main cause of the QEE leading to increased complications through Coincidences of Social Dynamics.
\end{abstract}

Keywords: Psychopathy; Amygdala; Ventromedial frontal cortex; Emotion; Careerist; Quantum entanglement entropy (QEE); Social Dynamics

\section{Introduction}

Psychopathy of the careerists is a disorder characterized by pronounced emotional deficits, marked by reduction in guilt and empathy, and involves increased risk for displaying antisocial behavior. The disorder is developmental. Psychopathic traits, particularly the emotional component, are relatively stable from childhood into adulthood [1]. One reason for the attention this classification receives is its strong predictive utility for institutional adjustment and recidivism (i.e., reoffending). Individuals with psychopathy are approximately three times more likely to reoffend than those with low psychopathic traits, and four times more likely to reoffend violently. Admittedly, it is the past antisocial behavior, indexed by psychopathy assessments, that is particularly important in predicting future criminal activity. However, it is the emotional component that characterizes psychopathy; high levels of antisocial behavior can develop from other neurobiological and socio-environmental risk factors. Psychopathy is not equivalent to the DSM-IV diagnosis of conduct disorder or antisocial personality disorder (ASPD) or their ICD-10 counterparts [2]. The psychiatric diagnoses focus on antisocial behavior rather than underlying causes; i.e., the emotion dysfunction seen in psychopathy. Individuals meeting the criteria for antisocial personality disorder are more heterogeneous in their pathophysiology than individuals meeting criteria for careerist psychopathy.

\section{Materials and Methods}

\section{Psychopathy as a careerist disorder of attention}

According to the response modulation hypothesis, the difficulty faced by individuals with psychopathy relates to a problem in reallocating attention to secondary information when engaged in goal-directed behavior. This difficulty in balancing the demands of goal-directed processing and secondary information processing creates a bias whereby psychopathic individuals are less responsive to affective information unless it is a central aspect of their goal-directed focus of attention. It is argued that "psychopathic individuals initially perceive and identify both primary and secondary information but are particularly adept at using higherorder processes to resolve the competition between goal-relevant and secondary demands on attention". The authors argue that these higher-order processes create an "early attention bottleneck" that limits the processing of secondary information. Typically, an early attention bottleneck has implied that only physical and not abstract properties of a secondary stimulus are processed; the 
bottleneck occurs within the visual stream, with "early" processing corresponding to physical feature as opposed to abstract feature processing [3]. However, Newman and colleagues use the term in a temporal sense; processing by higher order processes of the first stimulus in a sequence of stimuli acts as a bottleneck for processing the second stimulus in a sequence [4].

It is clear that regions implicated in top-down attentional control (i.e., higher order attentional processes), such as lateral frontal, dorsomedial, and parietal cortices, impact the amygdala's response to emotional stimuli. Increased priming of task-relevant representations by these regions is thought to reduce the representational strength of emotional stimuli within temporal cortex, following representational competition, and consequently reduce amygdala responses to these stimuli. In short, the reduced emotional responsiveness of individuals with elevated psychopathic traits could be a secondary consequence of heightened top-down attentional control to non-emotional stimulus features.

In recent studies in adults with psychopathy, Newman and colleagues manipulated attention either towards the threat-relevant component of a stimulus array or away from this component and examined fear-potentiated startle (FPS). In each of these studies, psychopathy scores were significantly inversely related to FPS under conditions that required participants to focus on a threatirrelevant dimension of stimuli. In contrast, psychopathy scores were unrelated to FPS when attention was focused on the threatrelevant dimension [5].

These studies provide important support for the suggestion that it is an attentional abnormality, rather than a problem in emotional responding, that is central to an understanding of psychopathy. Given the literature on the interaction of top-down attentional control and emotional responding, these data should suggest that psychopathy is related to enhanced recruitment of regions implicated in top-down attentional control (i.e., dorsomedial and lateral frontal and parietal cortices). The stronger these are recruited (as a function of psychopathy), the stronger the priming of threat irrelevant stimulus dimensions, the weaker the representation of threat relevant stimulus dimensions following representational competition, and the weaker the emotional response [6].

Emotion-based accounts of the careerist

Adults with psychopathy show a variety of emotional processing impairments. For example, they show:

(i) Reduced autonomic responses to the pain and distress of others

(ii) Reduced recognition of emotional expressions (for metaanalytic reviews of this literature, see refs 35,36 ). Interestingly, this impairment is relatively selective. Recognition of fearful, sad, and happy expressions is clearly reduced, while the recognition of disgusted and angry expressions remains intact

(iii) Reduced aversive conditioning; they are less likely to show autonomic activity to stimuli associated with shock

(iv) Pronounced difficulties with reinforcement-based decisionmaking.

These are seen in:

(a) Extinction: where the participant learns to respond to a stimulus for reward but, after a set number of trials, this responding must be extinguished because the reinforcement contingencies have changed, and the response is no longer rewarded

(b) Reversal learning: where the participant learns to make one form of response to a pair of stimuli to gain a reward but, after a set number of trials, this response must change, due to a change in reinforcement contingency, in order to gain the reward

(c) Economic decision-making paradigms: The Ultimatum game involves the participant and another individual. The participant has to decide whether to accept the offer of a share of resources made by the individual. This can be fair (eg, making a 50:50 split on $\$ 20$ so each gain $\$ 10$ ) or progressively unfair (eg, only $\$ 4$ is offered to the participant). Individuals with psychopathy show increased rejection of unfair offers, even at cost to themselves, relative to comparison individuals

(d) Moral judgment: Individuals with psychopathy show reduced responsiveness to what can be termed "care-based" transgressions (i.e., transgressions involving harm to another; eg, one person hitting another). This has been seen using a variety of paradigms. Again, this impairment in transgression processing is selective. Care-based transgressions are reliant on appropriate responsiveness to the pain and distress of others. As noted above, this is dysfunctional in adults with psychopathy. In contrast, conventional transgressions (reliant on authority; eg, talking in class) are reliant on appropriate responsiveness to other individual's anger while disgust-based transgressions (that can cover aspects of sexual behavior) are reliant on appropriate responsiveness of another individual's disgust.) Adults with psychopathy show intact processing of these emotional expressions. They also show intact processing of these forms of transgression. Given these data, a variety of authors have suggested that an emotional dysfunction underpins the deficits seen in individuals with psychopathy [7]. The oldest of these positions suggested that punishment processing was dysfunctional while reward processing was intact or even possibly superior. It is now clear that the situation is considerably more complicated.

Three critical data points are important to note. First, the expression impairment is not seen for all aversive expressions; it is seen for fear and sadness but not anger and disgust. This is inconsistent with a general impairment in processing aversive stimuli. Second, the expression processing impairment is also seen for happy expressions. This suggests that the processing of rewarded stimuli is also disrupted. Third, the immediate response to punishment is intact in adults with careerist psychopathy. 


\section{Structural magnetic resonance imaging (sMRI) studies of careerist}

A series of findings, reported across labs where appropriate IQ comparisons have been made, are worth noting. Not all studies have reported reduced volumes in these regions in psychopathy but none (at least involving IQ matched samples) have reported increased volumes in these regions. Thus, three studies have reported reduced amygdala volumes in adults with psychopathy including the largest structural imaging study of this population to date $(\mathrm{N}=296)$. Similarly, four studies have reported reductions in temporal pole and two in STS. Three studies have reported reductions in orbitofrontal cortex. Moreover, and interestingly given the extensive connections between the amygdala and orbitofrontal cortex though the uncinate fasciculus white matter tract, all three DTI studies examining the structural integrity of this tract in individuals with psychopathy have reported reduced structural integrity relative to comparison individuals.

\section{Functional magnetic resonance imaging (fMRI) studies of careerist}

Regrettably, again, many of the functional MRI (fMRI) studies of psychopathy, even when IQ was assessed (as an IQ<80 was exclusory), did not report that groups were matched for IQ making their interpretation problematic. Consequently, the data from such studies will not be considered here. However, studies where appropriate IQ matching was conducted include investigations of moral judgment, expression processing, emotional memory, processing abstract and concrete words, emotional Theory of Mind, and connectivity mapping.

Several of these studies support the sMRI findings of core dysfunction in the amygdala. Thus, individuals with psychopathy have been reported to show amygdala activity during moral judgment and also a weaker positive association between amygdala activity and severity ratings of transgressions than is seen in healthy individuals. In addition, violent schizophrenic patients with psychopathy show reduced amygdala responses to fearful expressions-though a relationship between psychopathic traits in aggressive individuals and amygdala responsiveness was not seen in another study. In addition, a reduction in amygdala activity was seen during an emotional memory paradigm. Currently, though the literature with respect to orbital/ventromedial frontal cortex (vmPFC) is less convincing. One study reported a reduction in the differential responsiveness of vmPFC to moral and nonmoral images. However, a second study reported increased vmPFC responses in individuals with psychopathy when performing a task involving the identification of other individual's emotional responsiveness (this increased vmPFC responsiveness did not relate to the emotional content as it was also seen in the nonemotional control condition). Notably, though, studies have shown weaker functional connectivity between vmPFC and the amygdala and between vmPFC and posterior cingulate cortex.

Given the findings of reductions in the temporal pole, it is interesting to note that studies have reported reduced responsiveness within this region to moral transgressions and abstract words. There have also been two reports of reduced posterior cingulate cortex responsiveness: during the processing of moral transgressions and emotional memory. In addition, posterior cingulate cortex shows reduced connectivity with vmPFC and regions of posterior cortex engaged in visual representation and attention priming. Two studies have also observed anomalous responsiveness within rostral medial frontal cortex. Thus, one study showed reduced activity within this region during moral judgment with increasing psychopathic traits. A second showed increased activity within this region relative to comparison individuals to judgments concerning vignette character's emotional states of careerist.

\section{Results}

Psychopathy of the careerists is a serious developmental disorder marked by pronounced emotional dysfunction and an increased risk for aggression. It is not equivalent to antisocial personality disorder from DSM-IV-R. Individuals meeting criteria for psychopathy with gold standard assessment techniques will also meet criteria for antisocial personality disorder. However, many other individuals with antisocial personality disorder will not meet criteria for psychopathy.

It is argued here that the emotion dysfunction relates to three core functional impairments: in the association of stimuli with reinforcement, the representation of expected value information and in prediction error signaling. These impairments are thought to relate to the observed dysfunction seen in both sMRI and fMRI studies within the amygdala, vmPFC, and (currently only in work with youth samples) striatum. Other regions of temporal cortex (temporal pole and superior temporal sulcus) may also be dysfunctional-though whether this reflects primary pathology or the secondary, developmental impact of dysfunction in the core regions is unclear. It is also unclear whether any functions reliant on these regions are detrimentally affected in careerist individuals with psychopathy.

Finally, there is sMRI and fMRI evidence of posterior cingulate cortex dysfunction. This is interesting given the extensive connectivity of this region with vmPFC and also its shared overlap in function. Both regions are implicated in the representation of expected value. However, as yet, no studies have formally investigated the representation of expected value within posterior cingulate cortex in adults with psychopathy of careerist.

Importantly, by specifying the computational and neural systems level impairments that are associated with this disorder, we now have available biomarkers of dysfunction. Such biomarkers are not only of potential use in diagnostic classification-the functional impairments in one aggressive patient may be very different from those of another-but also for assessing treatment efficacy. Currently, this disorder is regarded as extremely difficult to treat. Moreover, treatment studies are difficult when the outcome measure may be reoffending or incidence of aggressive episodes. However, with appropriate biomarkers it becomes possible to use 
these to determine treatment efficacy. The field is currently at this exciting stage. Now we need to identify effective treatments $[8,9]$.

A study of office politics suggests that workplaces are a jungle of awkward personalities vying for domination. Oliver James, the psychologist and broadcaster, identified three types of dysfunctional personalities among white collar workers: psychopath, Machiavellian, and narcissist [7].

These are the colleagues who have no compunction about trampling over others, or like nothing more than to plot and scheme, or who drone on endlessly about themselves. Most terrifyingly, the author concluded that there was fourth dysfunctional type: a "triadic person" who is a combination of all three. Such staff, James warns, have a dangerous, yet effective mix of a lack of empathy, selfcenteredness, deviousness and self-regard which can propel them to the top of the organizations.

Research has suggested that there has been an increase in the "triadic" conditions over the past 30 years because of changes in Western society and especially the rise of workplaces where there are no objective criteria for success or failure.

In a book, Office Politics, James warns how people who do not suffer from the disorders can lose out in the world of work and damage their emotional health unless they learn how to survive among such personalities. The warning may resonate among the almost eight million Britons who work in offices, as well as those based in schools, hospitals and particularly television studiosJames says broadcasting is infested with "triadic personalities".

Describing psychopathic tendencies, Machiavellian cunning and narcissistic selfishness a "dark triad", James says: "This dark triad of characteristics is very likely to be present in that person in your office who causes you so much trouble. Whether you work in the corporate sector, a small business or a public sector job, the system you are in is liable to reward ruthless, selfish manipulation.

James adds: "Television is jam-packed with untalented people who have managed to associate themselves with successful programmers and disassociate themselves from failures."

How to tell if your boss is psychopathic, Machiavellian, a narcissist or - even worse - all three.

For each character trait decide whether you strongly agree, agree, feel it applies sometimes, disagree or strongly disagree and give a score from 5 for strongly agree to 1 for strongly disagree. The higher the score, the more they have combined psychopathic, Machiavellian and narcissistic tendencies.

1. They tend to exploit and trick others for self-advancement

2. They have used lies and deception to get their way.

3. They have used ingratiation to get their way.

4. They tend to manipulate others for selfish reasons

5. They tend not to feel regretful and apologetic after having done wrong.
6. They tend not to worry about whether their behavior is ethical.

7. They tend to be lacking in empathy and crassly unaware of the distress they can cause others.

8. They tend to take a pretty dim view of humanity, attributing nasty motives and selfishness.

9. They tend to be hungry for admiration.

10. They tend to want to be the centre of attention.

11. They tend to aim for higher status and signs of their importance.

12. They tend to take it for granted that other people will make extra efforts to help them.

* Courtesy of Office Politics by Oliver James (Vermillion)

\section{Mechanism of psychopats metastases caused by the QEE}

Sporadic colon cancer is caused predominantly by dietary factors. We can select bile acids since high levels of hydrophobic bile acids accompany a Western-style diet and play a key role in colon carcinogenesis. Bile acid-induced stresses cause cell death in susceptible cells, contribute to genomic instability in surviving cells, impose Darwinian selection on survivors and enhance initiation and progression to colon cancer. The most likely major mechanism by which hydrophobic bile acids induce stresses on cells is the Quantum Entanglement Entropy (QEE) metastases through the DNA damage, endoplasmic reticulum stress, and mitochondrial damage. Persistent exposure of colon epithelial cells to hydrophobic bile acids can result by QEE in the activation of pro-survival stress-response pathways, and the modulation of genes/proteins associated with chromosome maintenance and mitosis. The mechanism of QEE by which hydrophobic bile acids contribute to genomic instability include oxidative DNA damage, p53 and other mutations, micronuclei formation and aneuploidy. Bile acids and oxidative stress decrease DNA repair proteins, an increase in DNA damage and increased genomic instability through this mechanism of metastases caused by Quantum Entanglement Entropy. This process provides a mechanistic explanation for the important QEE link between a Western-style diet and associated increased levels of colon cancer [10-16].

\section{Dichotomous correlations of career adaptation}

One prevalent description of translational medicine, first introduced by the Institute of Medicine's Clinical Research Roundtable, highlights two roadblocks (i.e., distinct areas in need of improvement): the first translational block (T1) prevents basic research findings from being tested in a clinical setting; the second translational block (T2) prevents proven interventions from becoming standard practice.

An important role in the processes of adaptation and masking in humans is playing also the immune system. The innate immune system functions as an interpreter of tissue damage and provides a 
first line of defense, also translates the information to other repair and defense systems in the body by stimulating angiogenesis, wound repair, and activating adaptive immunity. It is appropriate to consider autophagy a means for programmed cell survival balancing and counter-regulating apoptosis. Autophagy seems to have a dichotomous role in tumorigenesis and tumor progression.

Two other attributes play a similarly paradox role. The first involves major reprogramming of cellular energy metabolism in order to support continuous cell growth and proliferation replacing the metabolic program that operates in most normal tissues. The second involves active evasion by cancer cells from attack and elimination by immune cells. This capability highlights the dichotomous correlations of an immune system that both antagonizes and enhances tumor development and progression [17].

Evidence began to accumulate in the late 1990s confirming that the infiltration of neoplastic tissues by cells of the immune system serves counter-intuitively to promote tumor progression [14].

\section{The bipolar nature of career: HYBRID, twofaced new main law of nature}

The quantum entanglement is a basis of twofaced reality in which we are living our lives. From this reality are outgoing also the science and healthcare too. Although metastasis is important for systemic correlations expansion (as in tumors), it is a highly dichotomous process, with millions of cells being required to disseminate to allow for the selection of cells-correlates aggressive enough to survive the metastatic cascade. To quantify the dynamics of metastasis of correlations development, we need look at the coincidences of metastases in terms of co-occurrence at every point of time [9].To quantify co-occurrence we can use the -correlation between dichotomous variables defined as: where is the number of co-occurrence at time $t$. Than i and j represent particular site of metastasis, $\mathrm{X}$ represents the primary correlations type. The pairwise correlations (coincidences) between metastasis network links for every primary correlations' types and lead to the correlation coefficient matrix.

The dichotomous correlations of the adaptation may be caused also by the Quantum Entanglement Relative Entropy as a measure of distinguishability between two quantum states in the same Hilbert space. The relative entropy of two density matrices and is defined as. When and are reduced density matrices on a spatial domain D for two states of a quantum field theory (QFT), implies that increases with the size of D. Than is the change in entanglement entropy across $\mathrm{D}$ as one goes between the states.

When the states under comparison are close, the positivity is saturated to leading order [16].

The problem of conventional adaptation may be given by a definition of static, deterministic world. The proliferative correlations lead to the resonances between the degrees of freedom. When we increase the value of energy, we increase the regions where randomness prevails. For some critical value of energy, chaos appears over time we observe the exponential divergence of neighboring trajectories. For fully developed chaos, the cloud of points generated by a trajectory leads to diffusion. Here we must as first formulate a new Main Natural Law: the HYBRID Quantum Entanglement Entropy (HQEE) [14]. Through above resonances the QEE is causing a metastasis of correlations, antagonistically intertwining (coincidences) all types of potentially conflicting interests in cancer.

\section{Conclusion}

Throughout history, psychopaths, sociopaths, narcissists, and assorted antisocial-personality-disordered individuals have ruled societies [10]. Psychopaths and sociopaths often exhibit glibness and superficial charm, have a grandiose sense of self-worth, are pathological liars, display extreme narcissism, are deceitful, cunning and manipulative, exhibit a lack of remorse or guilt, show a callous disregard for the feelings of others, have no conscience, lack empathy, and fail to accept responsibility for their actions.

In a Careeristic Competitive World, the people who act immorally, who have no regard for truth, are going to have an advantage over those who play by the rules. The result is that those who achieve positions of power will be the most ruthless, the most sociopathic, the ones without conscience. In societies run by psychopaths, ambitious individuals and sycophants, who are not clinically psychopathic, are induced to model themselves after powerful psychopaths in order to achieve power. The result: psychopaths breed more psychopaths.

When corporate leaders, bankers, media executives, academics, military officers, government officials, Congressmen and Senators may be liars and deceivers, ruthless, callous manipulators who have no regard for truth or other people, the entire fabric of society is twisted in their image, and psychopathic behavior of careerists becomes the norm.

Our world is characterized by permanent war, and tendency to full-spectrum dominance in Global Government, and the New World Order. It is clear that this world has not been built by caring human beings but has been constructed and is run by powerful hallow soulless individuals. And, despite the violence, suffering and chaos they have caused, we allow them to remain at the top, and in control.

Knowledge of recent neurobiology is proving our thesis that Charles Darwin was wrong when formulated his theorem „Survival of the fittest ". It was the biggest false myth of the modern science. As we have demonstrated in our above study, the careerist is psychopat and not "the fittest “. From this reason we must to correct Charles Darwin to „Survival of the careerist “. Reality in 21 centuries is showing that Survival of the careerist based on the Quantum Entanglement Entropy (QEE) is more valid principle of Social Dynamics in our days. Careeristic Competition is the main cause of the QEE leading to increased complications through Coincidences of Social Dynamics. 


\section{Acknowledgments}

The author reports no competing interests. This work was supported by Dr. Marta Ballová, Ing. Jozef Balla, Ing. Konrad Balla and Livuska Ballová

\section{Conflict of Interests Disclosure}

The author declares no conflict of interests.

\section{References}

1. Cicchetti D, Dawson G (2002) Multiple levels of analysis. Dev Psychopathol. 14:417-420.

2. Koenigs M, Baskin Sommers A, Zeier J, Newman JP (2011) Investigating the neural correlates of psychopathy: a critical review. Mol Psychiatry 16(8): 792-799.

3. Newman JP, Curtin JJ, Bertsch JD, Baskin Sommers AR (2010) Attention moderates the fearlessness of psychopathic offenders. Biol Psychiatry 67(1):66-70.

4. Baskin Sommers AR, Curtin JJ, Newman JP (2011) Specifying the attentional selection that moderates the fearlessness of psychopathic offenders. Psychol Sci 22(2):226-234.

5. Hare RD (2003) Hare Psychopathy Checklist-Revised (PCL-R). 2nd Ed. Toronto, Canada: Multi Health Systems.

6. Blair RJR, Mitchell DGV, Blair KS (2005) The Psychopath: Emotion and the Brain. Oxford, UK: Blackwell.

7. Lynam DR, Caspi A, Moffitt TE, Loeber R, Stouthamer-Loeber M (2007) Longitudinal evidence that psychopathy scores in early adolescence predict adult psychopathy. J Abnorm Psychol 116(1):155-165.
8. Walters GD (2003) Predicting institutional adjustment and recidivism with the psychopathy checklist factor scores: a metaanalysis. Law Hum Behav 27(5): 541-558

9. Hemphill JF, Hare RD, Wong S (1998) Psychopathy and recidivism: a review. Legal Criminol Psychol 3: 139-170.

10. Skopec R I (2017) An Explanation of Biblic Radiation: Plasma. Journal of Psychiatry and Cognitive Behavior.

11. Skopec R II (2018) Artificial hurricanes and other new Weapons of Mass Destruction. International Journal of Scientific Research and Management 5(12): 7751-7764,201.

12.Skopec R III (2015) Intelligent Evolution, Complexity and SelfOrganization. NeuroQuantology 13: 299-303.

13. Skopec R IV (2016) Translational Biomedicine and Dichotomous Correlations of Masking. Translational Biomedicine 7(1): 47.

14. Skopec RV (2018) All Humans are Pre-Programmed to Innate Carcinogenesis through the Co-Occurrence of Metastases Caused by Quantum Entanglement Entropy. Archives of Oncology and Cancer Therapy 1(2): 29-36.

15. Skopec R VI (2019) Naphazoline Nitrate Treat the Frey Effect of Microwave and Other Sonic Weapon's Damages in Human's Internal, Endogenous Organs. International Journal of Research in Medical and Basic Sciences 5(1): 28-38.

16. Blair RJR (2007) The amygdala and ventromedial prefrontal cortex in morality and psychopathy. Trends Cogn Sci 11(9): 387-392.

17. Munoz LC, Frick PJ (2007) The reliability, stability, and predictive utility of the self-report version of the Antisocial Process Screening Device. Scand J Psychol 48(4):299-312 